\title{
Correction to "Dexamethasone-Induced Liver Enlargement Is Related to PXR/YAP Activation and Lipid Accumulation but Not Hepatocyte Proliferation"
}

\author{
The above article [Jiao T, Yao X, Zhao Y, Zhou Y, Gao Y, Fan S, Chen P, Li X, Jiang Y, Yang X, Gonzalez \\ FJ, Huang M, and Bi H (2020) Drug Metab Dispos, 48: 830-839; DOI: https://doi.org/10.1124/dmd.120. \\ 000061] was published with several errors introduced by the compositor: \\ The article should have carried a US government copyright line. \\ The article should have noted that it was funded in part by the Intramural Research Program of the National \\ Institutes of Health National Cancer Institute.
}

A Note Added in Proof was missing.

The PDF and HTML versions of the article have been corrected.

The compositor apologizes for any inconvenience caused by these errors. 